\title{
Azimuthal Jet Tomography at RHIC and LHC
}

\author{
Barbara Betz $^{\mathrm{a}}$, Miklos Gyulassy ${ }^{\mathrm{b}}$ \\ ${ }^{a}$ Institute for Theoretical Physics, Johann Wolfgang Goethe-University, 60438 Frankfurt am Main, Germany \\ ${ }^{b}$ Department of Physics, Columbia University, New York, 10027, USA
}

\begin{abstract}
Results based on a generic jet-energy loss model that interpolates between running coupling pQCD-based and AdS/CFT-inspired holographic prescriptions are compared to recent data on the high- $p_{T}$ pion nuclear modification factor and the high- $p_{T}$ elliptic flow in nuclear collisions at RHIC and LHC. The jet-energy loss model is coupled to various (2+1)d (viscous hydrodynamic) fields. The impact of energy-loss fluctuations is discussed. While a previously proposed AdS/CFT jet-energy loss model with a temperature-independent jet-medium coupling is shown to be inconsistent with the LHC data, we find a rather broad class of jet-energy independent energy-loss models $d E / d x=\kappa(T) x^{z} T^{2+z}$ that can account for the current data with different temperaturedependent jet-medium couplings $\kappa(T)$ and path-length dependence exponents of $0 \leq z \leq 2$.
\end{abstract}

Keywords: Jet Quenching, Viscous Hydrodynamics, Jet Holography

\section{Introduction}

Jet-quenching observables are considered as significant probes of the medium evolution during a heavy-ion collision. Below, we investigate a generic jet-energy loss model coupled to different temperature fields of the quark-gluon plasma (QGP) that interpolates between running coupling pQCD-based models and AdS/CFT-inspired holographic prescriptions. The results are compared to recent data on the nuclear modification factor $R_{A A}$ and the high- $p_{T}$ elliptic flow $[1,2]$, examining the dependence on the transverse momentum, the azimuthal angle, the centrality, and the collision energy to study the jet-medium coupling $\kappa$ and the impact of the jet path-length dependence. The latter one is usually considered to differentiate pQCD-based and AdS/CFT-inspired prescriptions with a linear $\left(\tau^{1}\right)$ and a squared $\left(\tau^{2}\right)$ path-length dependence, respectively.

The generic energy loss model studied is parametrized as

$$
\frac{d E}{d x}=\frac{d E}{d \tau}\left(\vec{x}_{0}, \phi, \tau\right)=-\kappa(T) E^{a}(\tau) \tau^{z} T^{c=2+z-a} \zeta_{q},
$$

with the jet-energy dependence, the path-length dependence, and the temperature dependence being characterized by the exponents $(a, z, c)$. The jet-medium coupling, $\kappa(T)$, may depend non-monotonically on the local temperature field, where $\kappa(T)=C_{r} \kappa^{\prime}(T)$ for quark $\left(C_{r}=1\right)$ and gluon $\left(C_{r}=\frac{9}{4}\right)$ jets. $T=T\left[\vec{x}(\tau)=\vec{x}_{0}+\left(\tau-\tau_{0}\right) \hat{n}(\phi)\right]$ describes the local temperature along the jet path at time $\tau$ for a jet initially produced time $\tau_{0}$.

The jets are distributed according to a transverse initial profile specified by the bulk QGP flow fields given by three variants of transverse plus Bjorken (2+1)d expansion [3]: (1) VISH2+1, (2) viscous RL hydro, and (3) a $v_{\perp}=0.6$ blast wave flow assuming radial dilation of the initial transverse profile.

We include skewed jet-energy loss fluctuations about the path-averaged mean by using a scaling factor $\zeta_{q}$ describing fluctuation distributions according to $f_{q}\left(\zeta_{q}\right)=\frac{(1+q)}{(q+2)^{1+q}}\left(q+2-\zeta_{q}\right)^{q}$. This class of distributions is controlled by 

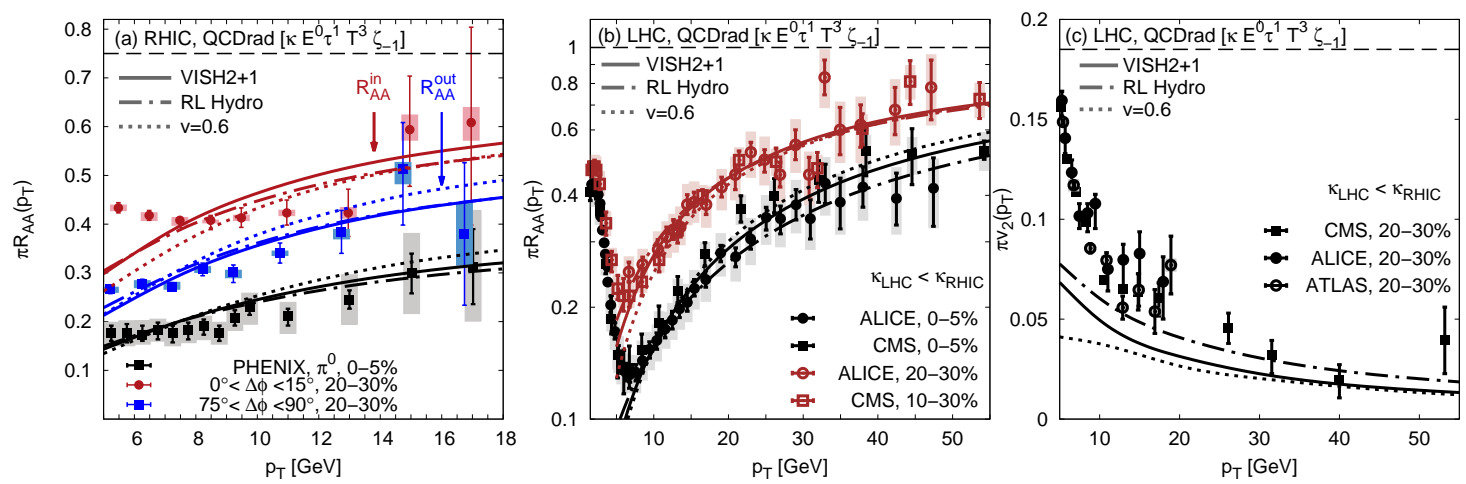

Figure 1. The nuclear modification factor in- and out-of-plane at RHIC (left panel) as well as the nuclear modification factor (middle panel) and the high- $p_{T}$ elliptic flow (right panel) at the LHC for a pQCD-based energy loss $d E / d \tau=\kappa E^{0} \tau^{1} T^{3} \zeta_{-1}$ without jet-energy loss fluctuations for various backgrounds [3] compared to the measured data [1,2].

the parameter $q>-1$ that interpolates between non-fluctuating $\left(q=-1, \zeta_{-1}=1\right)$, uniform Dirac distributions and distributions increasingly skewed towards small $\zeta_{q}<1$ for $q>-1$, similar to pQCD based models [4].

The above jet-energy loss prescription allows for an easy comparison of perturbative QCD (pQCD) based models with exponents $(0,1,3, q)[4]$ and conformal AdS holography models with non-linear path length $(0,2,4, q)[4,5]$, as well as phenomenological models based on a $\kappa(T)$ and $\kappa(\phi)[4]$ as discussed below.

\section{Results}

Fig. 1 shows the results for a pQCD-based model $(0,1,3,-1)$ without energy-loss fluctuations for the three different transverse backgrounds mentioned above both at RHIC (left panel) and at LHC energies (middle and right panel). Please note that the $R_{A A}$ in- and out-of-plane $\left[R_{A A}^{\text {in } / \text { out }}=R_{A A}\left(1 \pm 2 v_{2}\right)\right]$ allows for a simultaneous prescription of the measured nuclear modification factor and the high- $p_{T}$ elliptic flow. Here, the elliptic flow is given by the size of the gap between the nuclear modification factor in- and out-of-plane.

Fig. 1 clearly reveals that the results for both hydrodynamic prescriptions reproduce the measured data within the uncertainties of the bulk space-time evolution, given by the initial conditions, the viscosity $(\eta / s)$, the intial time $\tau_{0}$, the freeze-out time $T_{f}$, etc. considered. For this pQCD-based model we account for a running coupling $[6,7]$ explaining the "surprising transparency" of the QGP at the LHC.
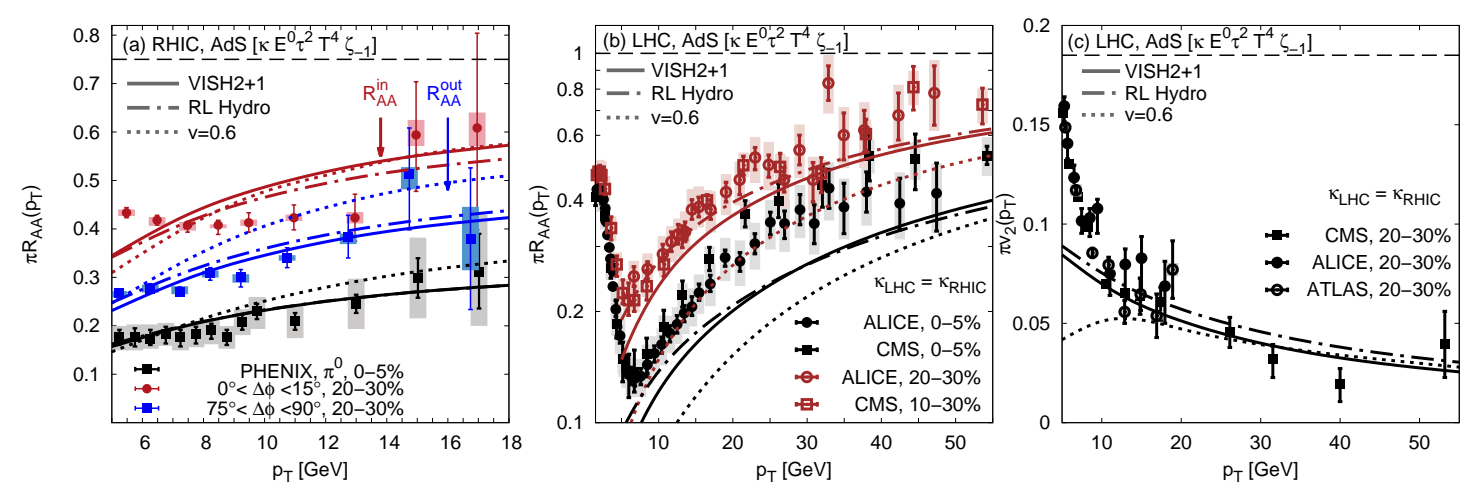

Figure 2. The nuclear modification factor in- and out-of-plane at RHIC (left panel) as well as the nuclear modification factor (middle panel) and the high- $p_{T}$ elliptic flow (right panel) at the LHC for an AdS/CFT-inspired energy loss $d E / d \tau=\kappa E^{0} \tau^{2} T^{4} \zeta_{-1}$ without jet-energy loss fluctuations for various backgrounds [3] compared to the measured data [1,2]. 

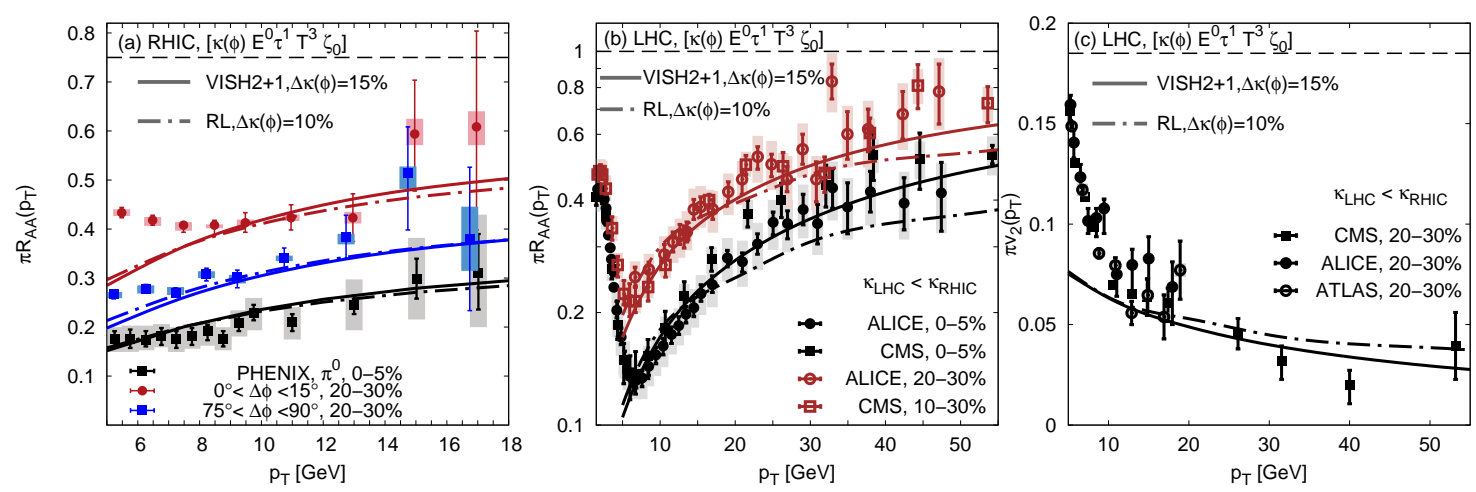

Figure 3. The nuclear modification factor in- and out-of-plane at RHIC (left panel) as well as the nuclear modification factor (middle panel) and the high- $p_{T}$ elliptic flow (right panel) at the LHC for a pQCD-based energy loss $d E / d \tau=\kappa(\phi) E^{0} \tau^{1} T^{3} \zeta_{0}$ with an azimuthal variation of the jet-medium coupling for two different hydrodynamic backgrounds [3] compared to the measured data [1,2].

Fig. 2 demonstrates that the conformal AdS/CFT-inspired prescription $(0,2,4,-1)$ without energy-loss fluctuations does certainly serve as a description of the measured data at RHIC energies but does not reproduce the measured $R_{A A^{-}}$ data at the LHC. In contrast, the jet-energy loss, shown in the middle panel of Fig. 2, is overpredicted. Please note that a conformal theory cannot have a running coupling, in contrast to the pQCD scenario considered in Fig. 1. If, however, a non-conformal AdS/CFT prescription is introduced, a reduction of the running-coupling constant with larger collision energies can be considered. This novel, non-conformal ansatz also describes both the nuclear modification factor and the high- $p_{T}$ elliptic flow measured at RHIC and at LHC for a (viscous) hydrodynamic background assuming about the same magnitude of reduction as for the pQCD prescriptions [4].

Moreover, Fig. 2(a) reveals that the background described by the simple blast wave model can also be ruled out as it usually leads to an elliptic flow that is too small as compared to data [4].

For pQCD, a reduction of the effective jet-medium coupling with $\sqrt{s}$ is natural due to the vacuum running of the perturbative QCD coupling $\alpha_{s}(Q)$. However, even after this reduction is taken into account, there is a tendency shown in Fig. 1(c) that the high- $p_{T}$ elliptic flow is underpredicted as compared to the measured data. This is in line with results of various pQCD-based models (AMY, HT, ASW, Molnar, CUJET2.0) $[1,7,8]$.

Ref. [7] suggested that there could well be an additional running of the running coupling constant w.r.t. the temperature, $\alpha_{\mathrm{eff}}(Q, T)$, as suggested by Lattice QCD [9]. As the temperature profiles in- and out-of-plane vary for a non-central collision, this might cause a modest $(10-15 \%)$ variation of the path-averaged coupling with a coupling constant enhanced out-of-plane. To simulate this effect, we study an azimuthal dependence of the jet-medium coupling by $\kappa(\phi)=\kappa \cdot(1+|\sin (\phi)| \cdot X)$, where $X$ is a given value in percentage.

Fig. 3 demonstrates that such a small azimuthal variation of $10-15 \%$ of the jet-medium coupling is sufficient to describe the $R_{A A}^{\text {in/out }}$ at RHIC as well as the $R_{A A}$ and the high- $p_{T} v_{2}$ at the LHC. Fig. 3 considers the pQCD-inspired prescription $(0,1,3,0)$ with energy-loss fluctuations. As shown in Ref. [4] (and Fig. 4), the jet-energy loss fluctuations themselves influence the yield of the nuclear modification factor but not the high- $p_{T}$ elliptic flow.

Please note that a moderate azimuthal dependence of the jet-medium coupling with a coupling enhanced out-ofplane describing the measured data supports a jet-medium coupling enhanced for lower temperatures [10] as a jet traversing out-of-plane will propagate longer through a comparably cooler medium.

In Fig. 4 we exemplary consider an exponentially falling ansatz for the jet-medium coupling, $\kappa(T)=\kappa_{1} e^{-b\left(T-T_{1}\right)}$, assuming that the coupling is zero below a certain temperature $T_{1}$, representing the freeze-out. At this $T_{1}$ the coupling peaks at a value of $\kappa_{1}$ and then falls off for larger temperatures to a value of $1 / e$ at a temperature $T_{e}$ [4]. Please note that this exponential drop of the jet-medium coupling implies an effective reduction of the jet-medium coupling at the LHC as compared to RHIC.

The results shown in Fig. 4 demonstrate that this exponential ansatz for the jet-medium coupling also describes the measured data within the present error bars even though the high- $p_{T}$ elliptic flow is again rather small. Surprisingly enough, the values of $T_{1}=160 \mathrm{MeV}$ and $T_{e}=200 \mathrm{MeV}$ indicate that the high-temperature medium is basically transparent $[4,6,7]$. 

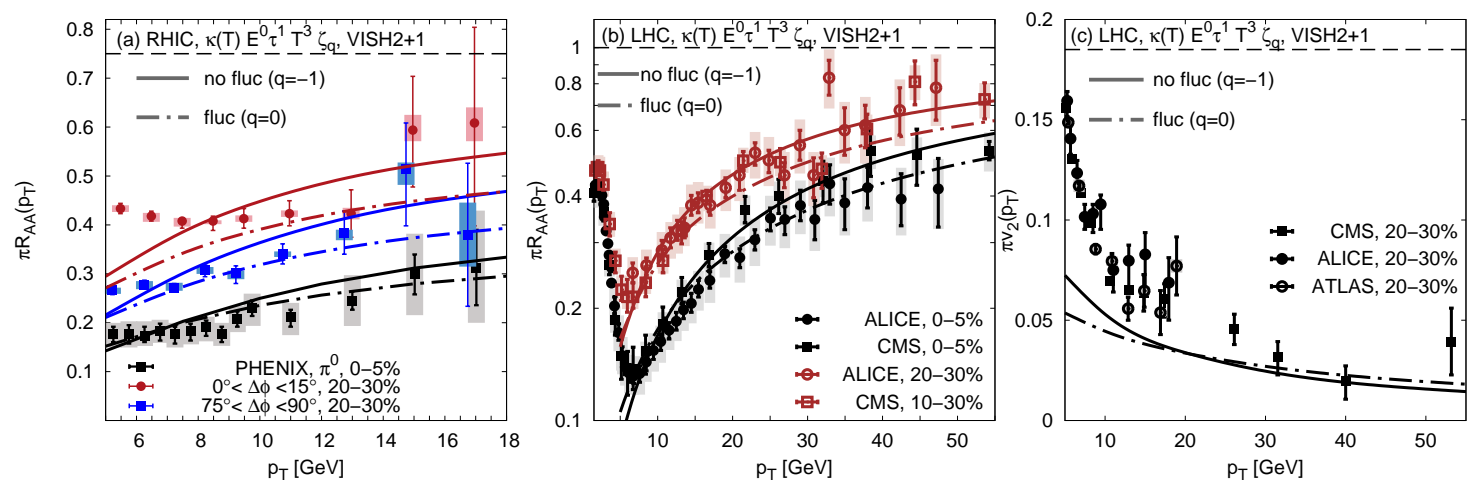

Figure 4. The nuclear modification factor in- and out-of-plane at RHIC (left panel) as well as the nuclear modification factor (middle panel) and the high- $p_{T}$ elliptic flow (right panel) at the LHC based on a pQCD-like energy loss $d E / d \tau=\kappa(T) E^{0} \tau^{1} T^{3} \zeta_{q}$ for a temperature-dependent $\kappa(T)=\kappa_{1} e^{-b\left(T-T_{1}\right)}$ (see text) with and without flucutations and a hydrodynamic background [3] compared to the measured data [1,2].

\section{Conclusions}

We compared the results of a generic jet-energy loss model, $d E / d \tau=-\kappa(T) E^{a} \tau^{z} T^{c=2+z-a} \zeta_{q}$, that interpolates between pQCD-based $(a=0, z=1, c=3)$ and AdS/CFT-inspired $(a=0, z=2, c=4)$ prescriptions to recent data $[1,2]$ on the nuclear modification factor and the high- $p_{T}$ elliptic flow both at RHIC and at LHC energies. The model discussed includes possible jet-energy loss fluctuations via the multiplicative factor $\zeta_{q}$. We found that the running coupling energy-loss models motivated by pQCD appears to be favored, while the AdS/CFT-inspired ansatz excluding by first principles the running of the coupling constant $\kappa$ cannot reproduce the measured data. We showed that the running of the coupling constant either with collision energy (Fig. 1) or with temperature (Fig. 4) reproduces the measured data within the present error bars considering (viscous) hydrodynamic background fields [3]. However, these prescriptions do not describe the high- $p_{T}$ elliptic flow with best accuracy which can only be reachted (Fig. 3) once an additional azimuthal dependence of the jet-medium coupling $[4,7]$ is considered with a coupling enhanced out-of-plane.

\section{Acknowledgement}

This work was supported in part through the Helmholtz International Centre for FAIR within the framework of the LOEWE program (Landesoffensive zur Entwicklung Wissenschaftlich-Ökonomischer Exzellenz) launched by the State of Hesse, the US-DOE Nuclear Science Grant No. DE-AC02-05CH11231 within the framework of the JET Topical Collaboration, and the US-DOE Nuclear Science Grant No. DE-FG02-93ER40764.

\section{References}

[1] A. Adare et al. [PHENIX Collaboration], Phys. Rev. C 87, 034911 (2013).

[2] B. Abelev et al. [ALICE Collaboration], Phys. Lett. B 720, 52 (2013); B. Abelev et al. [ALICE Collaboration], Phys. Lett. B 719, 18 (2013); S. Chatrchyan et al. [CMS Collaboration], Eur. Phys. J. C 72, 1945 (2012); S. Chatrchyan et al. [CMS Collaboration], Phys. Rev. Lett. 110, 042301 (2013); G. Aad et al. [ATLAS Collaboration], Phys. Rev. C 86, 014907 (2012); Phys. Lett. B 707, 330 (2012).

[3] H. Song and U. W. Heinz ("VISH2+1"), Phys. Rev. C 77, 064901 (2008); H. Song and U. W. Heinz ("VISH2+1"), Phys. Rev. C 78, 024902 (2008); C. Shen, U. Heinz, P. Huovinen and H. Song ("VISH2+1"), Phys. Rev. C 84, 044903 (2011); Z. Qiu, C. Shen and U. Heinz ("VISH2+1"), Phys. Lett. B 707, 151 (2012); C. Shen, U. Heinz, P. Huovinen and H. Song ("VISH2+1"), Phys. Rev. C 82, 054904 (2010); M. Luzum and P. Romatschke ("RL"), Phys. Rev. C 78, 034915 (2008); [Erratum-ibid. C 79, 039903 (2009)]; Phys. Rev. Lett. 103, 262302 (2009); M. Gyulassy, I. Vitev and X. N. Wang, Phys. Rev. Lett. 86, 2537 (2001).

[4] B. Betz and M. Gyulassy, JHEP 08 (2014) 090.

[5] C. Marquet and T. Renk, Phys. Lett. B 685, 270 (2010); S. S. Gubser et al., JHEP 0810, 052 (2008).

[6] B. Betz and M. Gyulassy, Phys. Rev. C 86, 024903 (2012); W. A. Horowitz and M. Gyulassy, Nucl. Phys. A 872, 265 (2011).

[7] J. Xu, A. Buzzatti and M. Gyulassy, JHEP 1408 (2014) 063.

[8] D. Molnar and D. Sun, arXiv:1305.1046 [nucl-th]; arXiv:1209.2430 [nucl-th]

[9] O. Kaczmarek, F. Karsch, F. Zantow and P. Petreczky, Phys. Rev. D 70, 074505 (2004) [Erratum-ibid. D 72, 059903 (2005)]

[10] J. Liao and E. Shuryak, Phys. Rev. Lett. 102, 202302 (2009); X. Zhang and J. Liao, Phys. Rev. C 89, 014907 (2014); Phys. Rev. C 87, 044910 (2013). 